\title{
Morning blood pressure surge: pathophysiology, clinical relevance and therapeutic aspects
}

This article was published in the following Dove Press journal: Integrated Blood Pressure Control

\author{
Grzegorz Bilo',2 \\ Andrea Grillo',2 \\ Valentina Guida ${ }^{1,2}$ \\ Gianfranco Parati ${ }^{1,2}$ \\ 'Department of Medicine and Surgery, \\ University of Milano-Bicocca, Milan, \\ Italy; ${ }^{2}$ Cardiology Unit, Department of \\ Cardiovascular, Neural and Metabolic \\ Sciences, San Luca Hospital, IRCCS \\ Istituto Auxologico Italiano, Milan, \\ Italy
}

\begin{abstract}
Morning hours are the period of the day characterized by the highest incidence of major cardiovascular events including myocardial infarction, sudden death or stroke. They are also characterized by important neurohormonal changes, in particular, the activation of sympathetic nervous system which usually leads to a rapid increase in blood pressure (BP), known as morning blood pressure surge (MBPS). It was hypothesized that excessive MBPS may be causally involved in the pathogenesis of cardiovascular events occurring in the morning by inducing hemodynamic stress. A number of studies support an independent relationship of MBPS with organ damage, cerebrovascular complications and mortality, although some heterogeneity exists in the available evidence. This may be due to ethnic differences, methodological issues and the confounding relationship of MBPS with other features of 24-hour BP profile, such as nocturnal dipping or BP variability. Several studies are also available dealing with treatment effects on MBPS and indicating the importance of long-acting antihypertensive drugs in this regard. This paper provides an overview of pathophysiologic, methodological, prognostic and therapeutic aspects related to MBPS.
\end{abstract}

Keywords: morning blood pressure surge, ambulatory blood pressure monitoring, cardiovascular risk, blood pressure variability

\section{Introduction}

Blood pressure (BP) level, whether assessed with conventional office measurements or with out-of-office techniques, is recognized as a major risk factor for cardiovascular and renal disease worldwide. ${ }^{1,2}$ Introduction of methods allowing BP monitoring prompted the search for new, potentially clinically relevant variables describing BP variations over time, in particular, over 24 hours. Among the numerous BP patterns and indices investigated, the behavior of BP in the morning has received significant interest. This is because morning hours are the period of the day characterized by the highest incidence of major cardiovascular events including myocardial infarction, sudden death or stroke. ${ }^{3}$ They are also characterized by important neurohormonal changes and, in most individuals, a rapid increase in $\mathrm{BP}^{4}$

It was hypothesized that the temporal relationship between morning BP surge (MBPS) and the morning peak of cardiovascular events might have a causal character and that MBPS might represent a new, independent cardiovascular risk factor. This hypothesis was supported by early works linking the size of MBPS with organ damage ${ }^{5}$ and cerebrovascular complications. ${ }^{6,7}$ This paper provides an overview of pathophysiologic, methodological, prognostic and therapeutic aspects related to MBPS.
Correspondence: Grzegorz Bilo Cardiology Unit, Department of Cardiovascular, Neural and Metabolic Sciences, San Luca Hospital, IRCCS Istituto Auxologico Italiano, Milan, Italy Tel +39026 I9II 2903

Email g.bilo@auxologico.it 


\section{Pathophysiology of MBPS}

Most physiological mechanisms of the body follow a circadian pattern, determined by a complex interaction of intrinsic biologic clock with environmental and behavioral factors. Morning hours are a critical period in this regard, with major changes in physiological control mechanisms occurring around the arousal time, driven by circadian system and modified by sensory inputs and posture. Many of these mechanisms have a direct effect on the cardiovascular system and contribute to BP increase. In particular, the changes in the activity of autonomic nervous system, mainly related to increased sympathetic activity, appear to be the key factor underlying MBPS.

The transition from sleep to waking is determined by the cortical arousal which is controlled by the "activating system" projecting to the thalamus and to the cerebral cortex. ${ }^{8}$ Many subcortical components act during cortical arousal. In particular, thalamic somatosensory neurons may be implied in the shift from the vagal to the sympathetic components of the autonomic nervous system. ${ }^{9}$ The release of corticotropicreleasing hormone which mediates the hypothalamicpituitary-adrenal axis and the autonomic components of responses to stressors contributes to the physiology of normal waking, ${ }^{10}$ and, along with the release of adrenocorticotropic hormone ${ }^{11}$ and of cortisol, ${ }^{12}$ is a potential mechanism in the increase of BP determined by the awakening process. An increase in secretion of epinephrine, testifying an increased sympathoadrenal activity, is found at the awakening, while the noradrenergic branches, determining the release of norepinephrine, are activated by the subsequent orthostasis. ${ }^{13}$

Circadian rhythms, the general importance of which has recently been acknowledged also by the Nobel Prize committee, play the key role in determining MBPS. A complex and reciprocal interplay exists between the autonomic, hormonal and behavioral components in determining the biologic variables displaying circadian fluctuations, including BP. Circadian rhythms of the cardiovascular system are coordinated by the suprachiasmatic nucleus of the hypothalamus, ${ }^{14}$ which receives inputs from the environment (such as light or temperature), from the cerebral cortex or from the body, integrates these signals and synchronizes autonomic output, hormone secretion and behavior. Activity of the suprachiasmatic nucleus, which is also feedback-regulated by the secretion of melatonin, is crucial in regulating the sympathetic and parasympathetic balance and in determining the effects of this balance to the heart and the vasculature and, therefore, the BP. ${ }^{15}$ The MBPS, which is mainly controlled by the sympathetic neural output to the cardiovascular system, ${ }^{16}$ may be, therefore, influenced by the synchronization and desynchronization of circadian clock.

While a moderate MBPS is a fully physiological phenomenon essential for maintaining adequate blood supply in a condition of increased activity, in some subjects, its entity appears to be excessive. The effector vascular mechanisms leading to excessive MBPS and its pathophysiological implications are not fully elucidated. Increased MBPS was shown to be associated with various factors, including aging, hypertension, abnormalities of glucose metabolism, alcohol intake, smoking, psychological and physical stress. ${ }^{17}$ Considering the role of circadian changes in sympathetic activity, ${ }^{18}$ studies showing that sympatholytic agents effectively lower morning BP in patients in whom it is poorly controlled support the relevance of changes in alpha-adrenergic vasoconstrictor activity in this regard. ${ }^{19-21}$ Sympathetic basal outflow, measured as muscle sympathetic nerve activity, seems unrelated to the MBPS in patients with hypertension. ${ }^{22}$ On the other hand, recent evidence suggests that the sympathetic "reactivity" triggered by an activation test, rather than the basal tone, may predict MBPS. ${ }^{23}$ In this context, a special role might be played by arterial baroreflex, which buffers the fluctuations in BP not only through its parasympathetic-mediated action on the heart rate (cardiac baroreflex) but also through modulating the sympathetic outflow to vasculature (sympathetic baroreflex). In fact, reduced sympathetic baroreflex was found to be associated with elevated MBPS both in the elderly ${ }^{24}$ and in healthy young individuals. ${ }^{25}$

The relationship between MBPS and baroreflex activity could be modified by stiffening of large arteries. The association between the MBPS and arterial stiffness was indeed observed, especially in elderly subjects, ${ }^{24}$ in whom the reduction of baroreflex sensitivity is particularly evident. This impairment of baroreflex could be explained by a reduced stretching of the arterial baroreceptors in the aortic arch and carotid arteries caused by increased stiffness of these large arteries. ${ }^{24,26}$ Moreover, stroke volume increase induced by neurohumoral changes occurring in the morning could directly favor a rapid increase of (mainly systolic) BP when the buffering capacity of large arteries is reduced due to increased stiffness. Considering more broadly this complex interplay, MBPS might thus be considered as a manifestation of the generally increased susceptibility of BP to undergo short-term fluctuations, reflected by measures of short-term BP variability. ${ }^{27} \mathrm{~A}$ recent study found that stiffness of large arteries is intrinsically associated with the MBPS, but this relationship seems to be mediated by an increased short-term $\mathrm{BP}$ variability, independently from mean BP values. ${ }^{28}$ 
While the autonomic nervous system seems to be the key player involved in MBPS, other neurohumoral factors which display circadian variations, such as the renin-angiotensin system $^{29}$ and the hypothalamic-pituitary-adrenal axis, could play a role. ${ }^{30}$ Although a link between these neurohormonal systems and the sympathetic activation is the most likely physiological explanation, ${ }^{31}$ specific studies regarding their role in the MBPS are lacking. While in normal subjects the pressor effect of these mechanisms could be counterbalanced by the vasodilating capacity of small arteries, in hypertensive individuals, small arteries remodeling might limit the capacity to buffer the increase in BP in the morning. ${ }^{32}$ Endothelial function could also be involved, considering that it is impaired in the morning even in normal subjects. ${ }^{33}$ Overall, the physiological background of increased MBPS is thus complex, and many intertwined mechanisms seem to be involved. Considering the association of this phenomenon with other unfavorable phenotypes (autonomic imbalance, arterial stiffness, BP variability, dysfunction of microcirculation), Kario proposed that MBPS might be a manifestation of the so-called systemic hemodynamic atherothrombotic syndrome, defined as a global alteration in circulatory function combining hemodynamic stress and vascular disease. ${ }^{34}$

The role of several environmental factors in determining MBPS was also postulated. In one study, MBPS was enhanced by salt loading in the non-salt-sensitive essential hypertension. ${ }^{35}$ Sympathetic activity might again be the crucial intermediate mechanism, given that its close link with salt intake was demonstrated in humans and in animal models, with a derangement in mechanisms of central sympathetic inhibition. ${ }^{36}$ The impact of differences in salt intake may underlie the observed ethnic differences in the degree and clinical determinants of MBPS (Figure 1), ${ }^{37-39}$ with higher MBPS found in Japanese compared with the Europeans, possibly being explained by higher salt intake in the former population. ${ }^{40}$ Weather and outdoor temperature might also influence both nighttime BP levels and the degree of the MBPS. ${ }^{41} \mathrm{~A}$ cold outdoor temperature and winter time are associated with the increased level of MBPS, ${ }^{42,43}$ as the levels of sympathetic activity are increased in response to low temperatures.

Finally, in treated hypertensive subjects, a sharp rise in BP in the morning hours could be caused by an inadequate antihypertensive regimen. As medications are often taken in the morning, short- or intermediate-acting compound could have insufficient effect in the hours prior to the administration of the next medication dose. In these cases, the time of administration of a specific drug could influence the control of BP at a specific time, for example, in the morning. ${ }^{44}$

Figure 2 summarizes the factors and mechanisms involved in the determination of MBPS.

\section{Assessment of MBPS}

MBPS is a dynamic feature of BP, strongly related with the subject's activity, and therefore, its appropriate definition and standardization of its assessment are of uttermost importance.

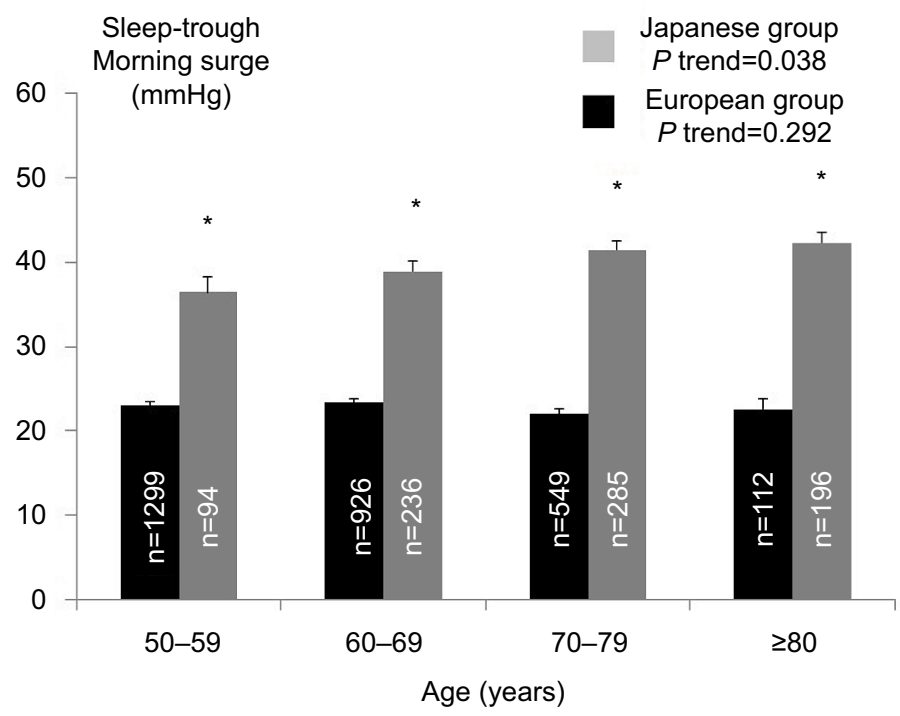

Figure I Sleep-trough morning SBP surge in two groups of Japanese (gray) and European (black) subjects, respectively.

Notes: Data adjusted for sex, body mass index, smoking, diabetes mellitus and 24-hour mean SBP. Data are separately shown for four different age groups. Values are expressed as means $\pm S E M$. $* P<0.001$ Japanese vs. European group in the same category. Hoshide $S$, Kario K, de la Sierra A, et al, Ethnic differences in the degree of morning blood pressure surge and in its determinants between Japanese and European hypertensive subjects novelty and significance, Hypertension, 20I5, 66, 750-756, http://hyper. ahajournals.org/. Promotional and commercial use of the material in print, digital or mobile device format is prohibited without the permission from the publisher Wolters Kluwer. Please contact permissions@lww.com for further information. ${ }^{37}$

Abbreviations: SBP, systolic blood pressure; SEM, standard error of the mean. 


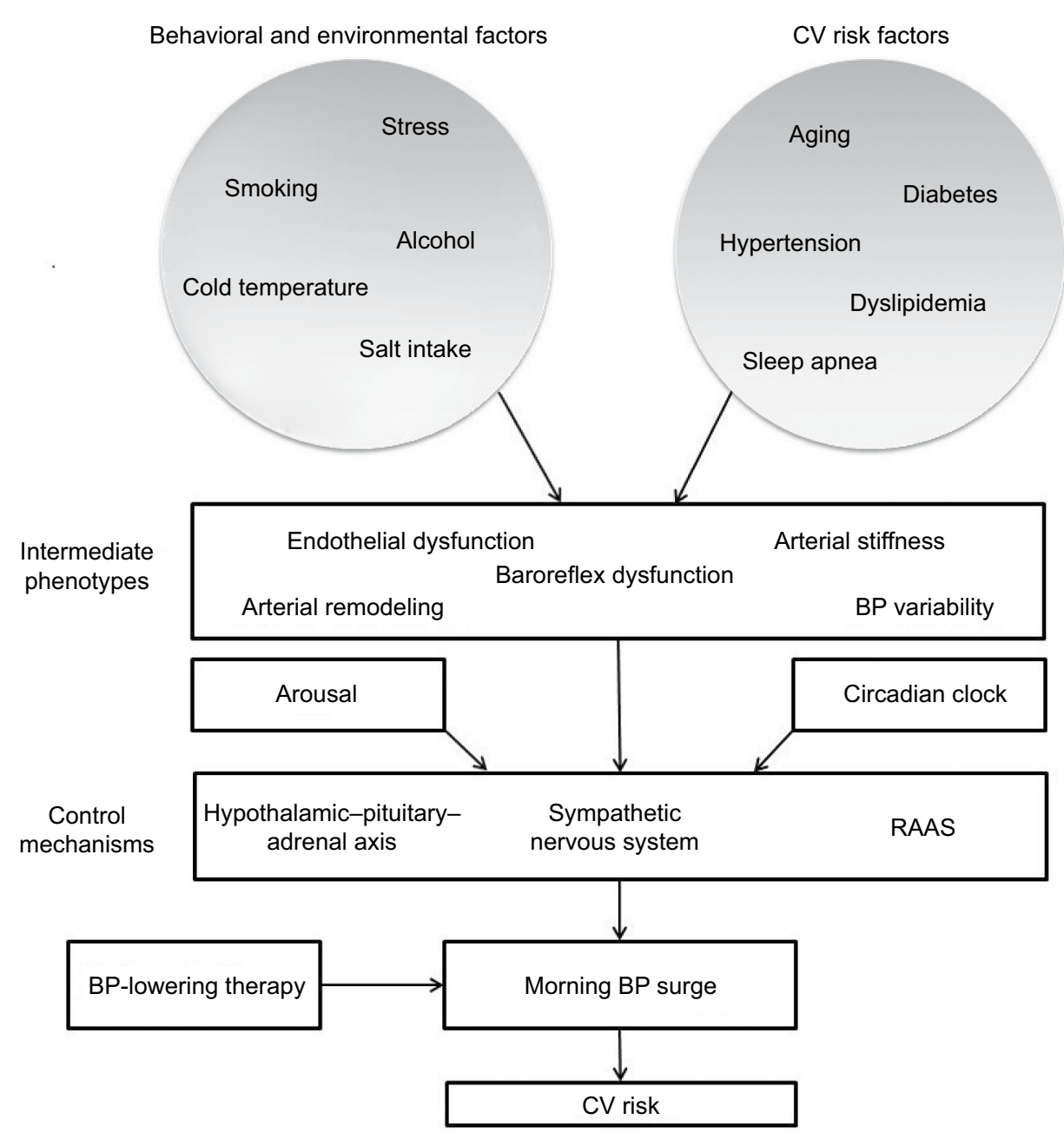

Figure 2 Pathophysiology of morning BP surge.

Abbreviations: $\mathrm{BP}$, blood pressure; $\mathrm{CV}$, cardiovascular; RAAS, renin-angiotensin-aldosterone system.

While several different definitions have been proposed by different groups, ${ }^{5,45-47}$ currently, most researchers refer to the definition proposed by Kario et al, ${ }^{6}$ according to which MBPS can be calculated as follows:

1. Sleep-trough MBPS, that is, the difference between the mean systolic BP (SBP) over 2 hours following the awakening and the average of three $\mathrm{BP}$ values centered on the lowest nocturnal BP

2. Prewaking MBPS, that is, the difference between the mean SBP over 2 hours following the awakening and the mean SBP over 2 hours preceding the awakening

It has to be acknowledged that even with these standardized definitions, the calculation of MBPS in individual subjects may create difficulties. In fact, some data indicate that the intra-individual reproducibility of MBPS estimates is poor. ${ }^{48}$ Some of the critical aspects are as follows: 1 ) proper identification of the awakening point - actigraphy is the most accurate solution, ${ }^{49}$ but information from a patient's logbook is easier to obtain and may be acceptable; 2) the impact of varying degrees of activity after awakening on MBPS;47 3) movement artifacts, common after awakening when the subject undertakes his/her morning activities and 4) in case of sleep-trough MBPS, the limited stability of trough BP estimate, driven by a single lowest BP measurement; moreover, if trough BP occurs early in the night, the sleep-trough MBPS does not fully represent the actual "surge" associated with the awakening. Some recent studies proposed that the assessment of MBPS slope rather than its size might represent a more stable and clinically relevant alternative to MBPS amplitude assessment. ${ }^{23,50}$

Apart from these methodological issues, there are also some physiological aspects of MBPS determination which may create interpretative difficulties not only in single subjects but also in research studies. This is because the size of MBPS is closely related to nocturnal BP fall (typically 
morning sleep BP difference is larger in "dippers", ie, individuals with a significant drop in nocturnal BP compared with daytime BP level). ${ }^{51-53}$ Also, enhanced BP variability may contribute to an increased $\mathrm{MBPS}^{28,53}$ - in particular, with larger BP variability, one can expect to find lower trough BP and, consequently, higher sleep-trough MBPS.

Finally, a universal cutoff for defining elevated MBPS is missing. Although Kario et al proposed $55 \mathrm{mmHg}$ cutoff, this value, representing the top decile of the population in study, cannot be readily generalized, considering that, for instance, in European subjects, MBPS is on average lower. ${ }^{6}$

By definition, MBPS assessment requires nighttime BP measurement, which currently can only be obtained with ambulatory BP monitoring. A different approach, achievable also with home BP monitoring, is based on the assessment of mean morning $\mathrm{BP}^{4}$ This static parameter is, however, rather different from a dynamic phenomenon such as MBPS and has different determinants.

\section{Prognostic relevance of MBPS}

The temporal relationship between MBPS and the peak incidence of cardiovascular events in the morning spurred the investigators to search for the possible cause-effect relationship with the hope of identifying in MBPS a new cardiovascular risk factor. Indirect data in this regard were provided by studies showing the association between MBPS and left ventricular hypertrophy, ${ }^{5}$ vascular inflammatory response and plaque instability. ${ }^{54,55}$ The first solid evidence on the relationship of MBPS with cardiovascular events became available in 2003 when Kario et al reported an independent association between MBPS and silent cerebral infarcts as well as with incident stroke, with hazard ratios for each $10 \mathrm{mmHg}$ increase in the sleep-trough and preawakening MBPS being 1.22 (95\% CI: 1.05-1.40) and 1.14 (95\% CI: 0.99-1.31), respectively. ${ }^{6}$ A few years later, a report from the Ohasama study was published indicating the association of MBPS with hemorrhagic stroke only. ${ }^{7}$

Further studies were performed subsequently providing somewhat discordant results: overall, the predictive role of MBPS was confirmed in Asian studies, while in several other populations, independent associations with outcomes were not found. ${ }^{51,53}$ The reasons behind these conflicting findings may be multiple. First, the possible role of ethnic differences (genetic and lifestyle related) was postulated in this regard. ${ }^{37,56}$ Second, the role of confounding influence of nocturnal BP fall was hypothesized. Italian researchers found that an independent association of MBPS with stroke in elderly hypertensive subjects was only present in those with preserved nocturnal fall (dippers), ${ }^{57,58}$ also if mean BP was well controlled. ${ }^{59}$ Third, the differences in the composition of populations among studies may be a relevant factor (general population vs. middle-aged hypertensives vs. elderly hypertensives).

Further insight into the possible factors determining the association of MBPS with prognosis is provided by the International Database of Ambulatory Blood Pressure Monitoring in Relation to Cardiovascular Outcomes data. In this multinational dataset, MBPS was associated with all-cause mortality and cardiac events, but, interestingly, not with cardiovascular mortality or cerebrovascular events (except hemorrhagic stroke in Asian subjects, a result probably driven by the inclusion of Ohasama data). Moreover, a number of these associations became evident only after adjusting for nocturnal BP fall. The association of MBPS with cardiovascular mortality appeared, somewhat surprisingly, to be closer in Europeans than in Asian participants. The relationship between MBPS and risk was not linear and worse outcome was evident only in the upper ethnic- and sex-specific decile of MBPS and in any case above $20 \mathrm{mmHg}$ for both sleep-trough and preawakening MBPS. ${ }^{60}$ The close association with noncardiovascular deaths in this study might suggest that MBPS, rather than being the cause of events, is an epiphenomenon indicating generally poor health conditions.

Most of the currently available evidence was summarized in the meta-analysis of Sheppard et al, which, however, did not produce conclusive results. The authors were unable to identify a significant association between elevated MBPS (as a categorical variable) and cardiovascular disease. When MBPS was considered as a continuous variable, a significant increase of stroke risk (by $11 \%$ per $10 \mathrm{mmHg}$ of MBPS increase) was observed. ${ }^{61}$ Also, another meta-analysis published in parallel by Xie et al was unable to show the association between large MBPS and cardiovascular outcomes. On the other hand, in line with the International Database of Ambulatory Blood Pressure Monitoring in Relation to Cardiovascular Outcomes data (included in this meta-analysis), a significant association with all-cause mortality was reported. ${ }^{62}$ Unfortunately, both these studies share the common limitations of observational data meta-analyses related to the major heterogeneity existing among studies in terms of outcomes, data reporting, variables used for adjustments and so on.

Finally, a recently published study in a general population sample in Taipei reported that a high rate (slope, ie, amplitude/time span), rather than the amplitude of MBPS, is associated with worse outcome. The authors found higher all-cause and cardiovascular mortality above 95 th percentile $(\geq 11.3 \mathrm{mmHg} / \mathrm{h})$, regardless of the presence or absence of 
morning hypertension. The results were supported by a simulation analysis indicating that MBPS rate may be a more stable and robust indicator of BP changes in the morning. ${ }^{50}$

A detailed overview of the evidence linking MBPS and morning BP with outcomes can be found in the recently published excellent review by Wang et al. ${ }^{4}$

\section{Pharmacologic treatment and MBPS}

The effects of BP-lowering treatment in the morning hours have two distinct (although not unrelated) aspects: the need for adequate control of BP levels in the morning and the impact of treatment on the dynamic BP change, that is, MBPS. The former issue is closely related to the duration of antihypertensive effect of drugs; given that antihypertensive therapy is commonly given in the morning, the loss of BP-lowering action in the final hours of dosing period in case of inadequate 24-hour coverage will inevitably lead to worsening of BP control in the morning hours, a frequent phenomenon among treated hypertensive patients. ${ }^{63}$ Moreover, a number of studies provided evidence that the use of longacting drugs may indeed improve BP control in the morning hours. ${ }^{64-67}$ Considering that the issue of hypertension control in the morning hours and of "isolated morning hypertension" has been recently extensively reviewed elsewhere, the following discussion will focus specifically on the effects of treatment on MBPS. ${ }^{4}$

Several factors might be involved in the determination of the effects of specific drugs on MBPS.

- Duration of BP-lowering action

Although for most currently used antihypertensive drugs, the reduction of BP-lowering effect during the hours preceding the next morning dose is gradual, it is possible that, in case of drugs providing an incomplete 24-hour coverage, a progressive decrease in drug concentration over the hours when MBPS is assessed (eg, between night-trough and postawakening) might contribute to a major morning BP increase.

- Relationship between BP level and variability

Worse control of BP in the final hours of dosing period with shorter acting drugs could also favor major short-time changes in BP (such as MBPS), in line with the known direct relationship between $\mathrm{BP}$ levels and $\mathrm{BP}$ variability. ${ }^{27}$

- Drug intake timing

Both the above mechanisms could be relevant when considering the "chronobiological" approach based on bedtime administration of antihypertensive drugs, especially the shorter acting ones. ${ }^{68,69}$

- Pharmacodynamic aspects

Some drug classes might be more active than others against the mechanisms driving MBPS.

While evidence on the effects of drugs on morning BP is quite abundant, fewer studies directly address the impact of specific drugs on MBPS. In a crossover trial performed in nondiabetic hypertensive patients, a long-acting betablocker, nebivolol, significantly lowered sleep-trough systolic MBPS from baseline with no significant difference between morning and evening administration (MBPS reduction by $7.5 \pm 18.2$ and $11.1 \pm 31.4 \mathrm{mmHg}$, respectively, $P=0.5) .{ }^{70}$ Rosito et al found a significant reduction in systolic MBPS with a nondihydropyridine calcium antagonist, verapamil, compared with placebo (MBPS on treatment $9.5 \pm 3.3$ vs. $19.7 \pm 3.6 \mathrm{mmHg}$, respectively, $P<0.04) .{ }^{46}$ Lack of active control from a different antihypertensive class in these studies did not allow concluding whether the observed effects were class specific. Such comparison was performed in the International Verapamil SR-Trandolapril (INVEST) Ambulatory Monitoring Substudy, which did not reveal significant differences in the size of MBPS between verapamil and beta-blocker atenolol in patients with coronary artery disease. $^{71}$

Another crossover study compared the effects of an angiotensin-converting enzyme inhibitor, lisinopril, with angiotensin receptor blocker (ARB), candesartan, in a single morning administration. The authors found a major reduction in MBPS with candesartan among subjects who had large MBPS at baseline, the difference being mainly driven by lower morning BP during candesartan treatment. ${ }^{72}$ Considering that both drugs are renin-angiotensin system antagonists, it is unlikely that the observed difference in MBPS was due to mechanism-specific effects. A plausible alternative explanation in this case is a shorter duration of BP-lowering effect reported for lisinopril than for candesartan (trough-to-peak ratios about 0.7 and 1.0, respectively). Similarly, in a study comparing long-acting calcium antagonist, amlodipine, with intermediate-acting ARB, valsartan, in monotherapy, only the former significantly reduced morning SBP, while both agents reduced the lowest night SBP to a similar extent. Consequently, the reduction in morning SBP surge was significantly greater in patients treated with amlodipine $(-6.1$ vs. $+4.5 \mathrm{mmHg}, P<0.02){ }^{66}$ Also, by comparing amlodipine with another intermediateacting ARB, losartan, a tendency in favor of amlodipine 


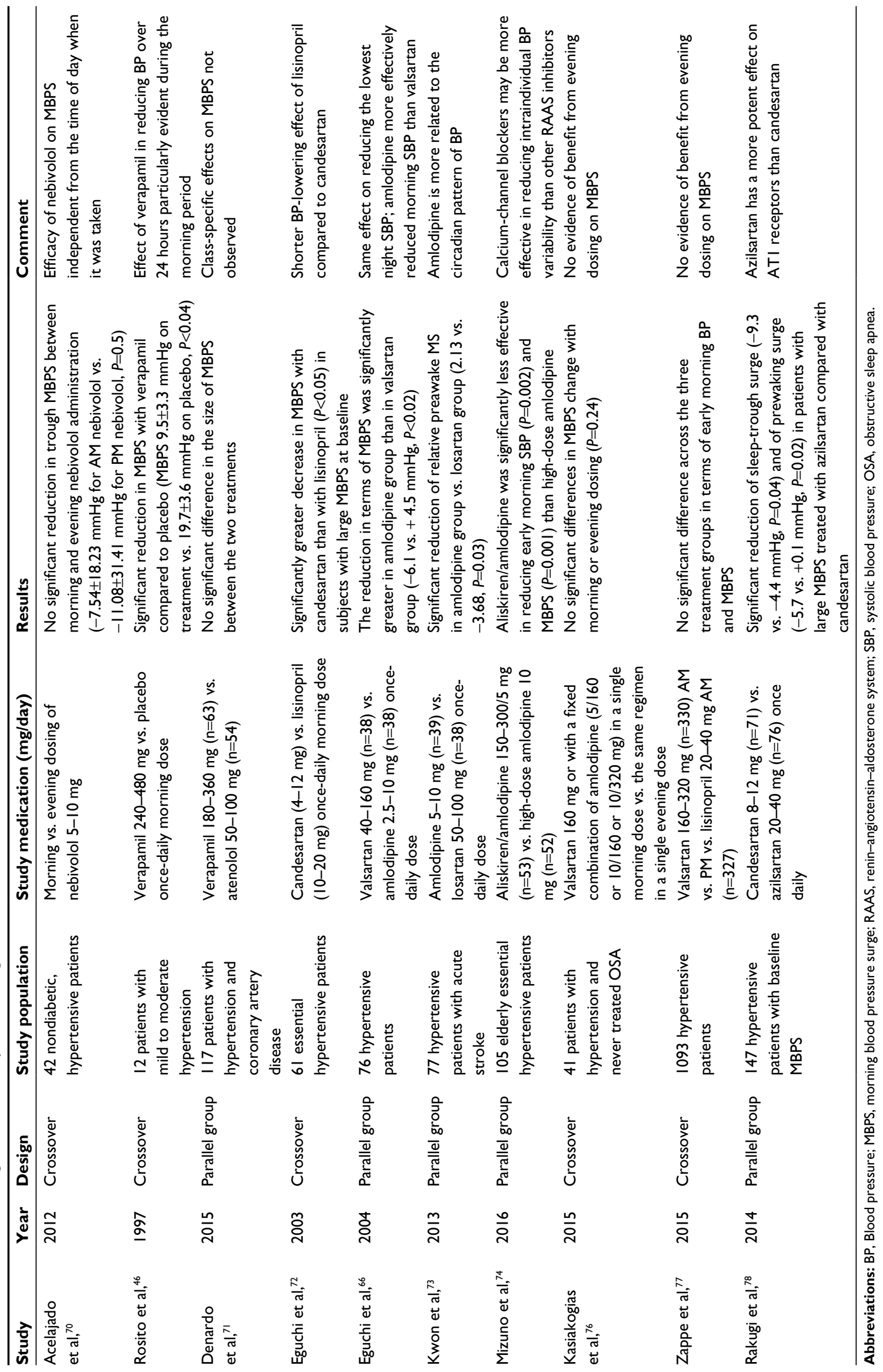


was observed. ${ }^{73}$ Interesting results were obtained in a study comparing high-dose $(10 \mathrm{mg})$ amlodipine monotherapy with aliskiren/amlodipine $(150 / 5 \mathrm{mg})$ association. In this study, the high dose of calcium antagonist controlled the morning BP and reduced MBPS better. ${ }^{74}$

Chronotherapeutic approach to hypertension treatment has received attention and several studies suggested possible benefits from bedtime dosing of antihypertensive drugs. ${ }^{75}$ Nonetheless, the available evidence does not indicate any clear benefit in terms of MBPS reduction with evening drug intake (Table 1). ${ }^{70,76,77}$

Apart from the duration of action, the antihypertensive efficacy of drugs may also be relevant. Rakugi et al compared two long-acting ARBs, candesartan and azilsartan, and found a significant MBPS reduction in subjects with large baseline MBPS who received azilsartan, a more potent AT1 receptor blocker. $^{78}$

Taken together, the available evidence suggests that the key element in controlling MBPS is the use of long-acting drugs, which provide effective coverage in the morning hours (Table 1), while there are no clear data supporting the use of any specific antihypertensive drug class to that aim. Studies demonstrating that MBPS reduction with treatment might improve clinical outcomes are also lacking.

\section{Conclusion}

MBPS is a complex phenomenon driven by several mechanisms, among which sympathetic activation plays the central role. It is closely related with a number of physiological variables, including arterial stiffness and endothelial function, as well as with specific patterns characterizing 24-hour BP profile, such as nocturnal BP fall and BP variability. Considering the limited reproducibility of current MBPS estimates, further research is needed to refine the methodology of its assessment.

While a number of studies demonstrated the association of MBPS with outcomes, it is not clear whether it should be considered a causal factor or only an epiphenomenon of global impairment of cardiovascular function. It is also not clear whether it may represent a target for treatment, although the available evidence suggests that MBPS size may be limited by strategies based on the use of long-acting drugs, aimed at providing a stable and sustained BP control throughout the 24 hours.

\section{Disclosure}

The authors report no conflicts of interest in this work.

\section{References}

1. Whelton PK, Carey RM, Aronow WS, et al. 2017 ACC/AHA/AAPA/ $\mathrm{ABC} / \mathrm{ACPM} / \mathrm{AGS} / \mathrm{APhA} / \mathrm{ASH} / \mathrm{ASPC} / \mathrm{NMA} / \mathrm{PCNA}$ guideline for the prevention, detection, evaluation, and management of high blood pressure in adults: a report of the american college of cardiology/american heart association task force on clinical Pr. J Am Coll Cardiol. Epub 2017 Nov 13.

2. Mancia G, Fagard R, Narkiewicz K, et al. 2013 ESH/ESC guidelines for the management of arterial hypertension. J Hypertens. 2013;31:1281-1357.

3. Muller JE. Circadian variation in cardiovascular events. Am J Hypertens. 1999; 12:35S-42S.

4. Wang J-G, Kario K, Park J-B, Chen C-H. Morning blood pressure monitoring in the management of hypertension. $J$ Hypertens. 2017;35:1554-1563.

5. Kuwajima I, Mitani K, Miyao M, Suzuki Y, Kuramoto K, Ozawa $\mathrm{T}$. Cardiac implications of the morning surge in blood pressure in elderly hypertensive patients: relation to arising time. Am J Hypertens. 1995;8:29-33.

6. Kario K, Pickering TG, Umeda Y, et al. Morning surge in blood pressure as a predictor of silent and clinical cerebrovascular disease in elderly hypertensives: a prospective study. Circulation. 2003;107: 1401-1406.

7. Metoki H, Ohkubo T, Kikuya M, et al. Prognostic significance for stroke of a morning pressor surge and a nocturnal blood pressure decline: the Ohasama study. Hypertension. 2006;47:149-154.

8. Åkerstedt T, Billiard M, Bonnet M, et al. Awakening from sleep. Sleep Med Rev. 2002;6:267-286.

9. Massimini M, Porta A, Mariotti M, Malliani A, Montano N. Heart rate variability is encoded in the spontaneous discharge of thalamic somatosensory neurones in cat. $J$ Physiol. 2000;526( Pt 2):387-396.

10. Chang FC, Opp MR. Corticotropin-releasing hormone (CRH) as a regulator of waking. Neurosci Biobehav Rev. 2001;25:445-453.

11. Born J, Hansen K, Marshall L, Mölle M, Fehm HL. Timing the end of nocturnal sleep. Nature. 1999;397:29-30.

12. Connell JM, Whitworth JA, Davies DL, Lever AF, Richards AM, Fraser R. Effects of ACTH and cortisol administration on blood pressure, electrolyte metabolism, atrial natriuretic peptide and renal function in normal man. J Hypertens. 1987;5:425-433.

13. Dodt C, Breckling U, Derad I, Fehm HL, Born J. Plasma epinephrine and norepinephrine concentrations of healthy humans associated with nighttime sleep and morning arousal. Hypertension. 1997;30: 71-76.

14. Buijs RM, Escobar C, Swaab DF. The circadian system and the balance of the autonomic nervous system. In: Handbook of Clinical Neurology. Edinburgh: Elsevier. 2013:173-191.

15. Scheer FA, Kalsbeek A, Buijs RM. Cardiovascular control by the suprachiasmatic nucleus: neural and neuroendocrine mechanisms in human and rat. Biol Chem. 2003;384:697-709.

16. Schofl C, Becker C, Prank K, et al. Twenty-four-hour rhythms of plasma catecholamines and their relation to cardiovascular parameters in healthy young men. Eur J Endocrinol. 1997;137:675-683.

17. Kario K. Morning surge in blood pressure and cardiovascular risk: evidence and perspectives. Hypertension. 2010;56:765-773.

18. Panza JA, Epstein SE, Quyyumi AA. Circadian variation in vascular tone and its relation to alpha-sympathetic vasoconstrictor activity. $N$ Engl $J$ Med. 1991;325:986-990.

19. Hashimoto J, Chonan K, Aoki Y, et al. Therapeutic effects of evening administration of guanabenz and clonidine on morning hypertension: evaluation using home-based blood pressure measurements. J Hypertens. 2003;21:805-811.

20. Gonokami K, Obara T, Kobayashi M, et al. Blood pressure-lowering effect and duration of action of bedtime administration of doxazosin determined by home blood pressure measurement. Clin Exp Hypertens. 2010;32:311-317. 
21. Kario K, Matsui Y, Shibasaki S, et al. An $\alpha$-adrenergic blocker titrated by self-measured blood pressure recordings lowered blood pressure and microalbuminuria in patients with morning hypertension: the Japan Morning Surge-1 Study. J Hypertens. 2008;26:1257-1265.

22. Hering D, Kucharska W, Kara T, Somers VK, Narkiewicz K. Resting sympathetic outflow does not predict the morning blood pressure surge in hypertension. J Hypertens. 2011;29:2381-2386.

23. Lambert EA, Chatzivlastou K, Schlaich M, Lambert G, Head GA. Morning surge in blood pressure is associated with reactivity of the sympathetic nervous system. Am J Hypertens. 2014;27:783-792.

24. Okada Y, Galbreath MM, Shibata S, et al. Morning blood pressure surge is associated with arterial stiffness and sympathetic baroreflex sensitivity in hypertensive seniors. AJP Hear Circ Physiol. 2013;305:H793-H802.

25. Johnson AW, Hissen SL, Macefield VG, Brown R, Taylor CE. Magnitude of morning surge in blood pressure is associated with sympathetic but not cardiac baroreflex sensitivity. Front Neurosci. 2016;10:412.

26. Grillo A, Lonati LM, Guida V, Parati G. Cardio-ankle vascular stiffness index (CAVI) and 24-h blood pressure profiles. Eur Hear J Suppl. 2017;19:B17-B23.

27. Parati G, Ochoa JE, Lombardi C, Bilo G. Assessment and management of blood-pressure variability. Nat Rev Cardiol. 2013;10:143-155.

28. Pucci G, Battista F, Anastasio F, Schillaci G. Morning pressor surge, blood pressure variability, and arterial stiffness in essential hypertension. J Hypertens. 2017;35:272-278.

29. Naito Y, Tsujino T, Fujioka Y, Ohyanagi M, Iwasaki T. Augmented diurnal variations of the cardiac renin-angiotensin system in hypertensive rats. Hypertension. 2002;40:827-833.

30. Kario K. Vascular damage in exaggerated morning surge in blood pressure. Hypertension. 2007;49:771-772.

31. Fisher JP, Paton JFR. The sympathetic nervous system and blood pressure in humans: implications for hypertension. J Hum Hypertens. 2012;26:463-475.

32. Rizzoni D, Porteri E, Platto C, et al. Morning rise of blood pressure and subcutaneous small resistance artery structure. J Hypertens. 2007;25:1698-1703.

33. Otto ME, Svatikova A, Barretto RB et al. Early morning attenuation of endothelial function in healthy humans. Circulation. 2004;109:2507-2510.

34. Kario K. Morning surge in blood pressure: a phenotype of systemic hemodynamic atherothrombotic syndrome. Am J Hypertens. 2015;28:7-9.

35. Osanai T, Okuguchi T, Kamada T, et al. Salt-induced exacerbation of morning surge in blood pressure in patients with essential hypertension. J Hum Hypertens. 2000;14:57-64.

36. Strazzullo P, Barbato A, Vuotto P, Galletti F. Relationships between salt sensitivity of blood pressure and sympathetic nervous system activity: a short review of evidence. Clin Exp Hypertens. 2001;23:25-33.

37. Hoshide S, Kario K, de la Sierra A, et al. Ethnic differences in the degree of morning blood pressure surge and in its determinants between Japanese and European hypertensive subjects novelty and significance. Hypertension. 2015;66:750-756.

38. Eguchi K, Hoshide S, Schwartz JE, Shimada K, Kario K. Visit-to-visit and ambulatory blood pressure variability as predictors of incident cardiovascular events in patients with hypertension. Am J Hypertens. 2012;25:962-968.

39. Lee DH, Ihm SH, Youn HJ, et al. Age is an independent risk factor for the early morning blood pressure surge in patients never-treated for hypertension. Korean Circ J. 2009;39:322-327.

40. Stamler J, Elliott P, Dennis B, et al. INTERMAP: background, aims, design, methods, and descriptive statistics (nondietary). J Hum Hypertens. 2003;17:591-608.

41. Modesti PA, Morabito M, Bertolozzi I, et al. Weather-related changes in 24-hour blood pressure profile: effects of age and implications for hypertension management. Hypertension. 2006;47:155-161.

42. Murakami S, Otsuka K, Kono T, et al. Impact of outdoor temperature on prewaking morning surge and nocturnal decline in blood pressure in a Japanese population. Hypertens Res. 2011;34:70-73.
43. Kario K. Caution for winter morning surge in blood pressure: a possible link with cardiovascular risk in the elderly. Hypertension. 2006;47:139-140.

44. Morgan TO. Should blood pressure medication be taken in the morning or evening? J Hypertens. 2015;33:263-265.

45. Shimada K, Kario K, Umeda Y, Hoshide S, Hoshide Y, Eguchi K. Early morning surge in blood pressure. Blood Press Monit. 2001;6:349-353.

46. Rosito GA, Gebara OC, McKenna CA, Solomon HS, Muller JE, Tofler GH. Effect of sustained-release Verapamil on the morning systemic arterial pressure surge during daily activity in patients with systemic hypertension. Am J Cardiol. 1997;79:1252-1255.

47. Leary AC, Struthers AD, Donnan PT, MacDonald TM, Murphy MB. The morning surge in blood pressure and heart rate is dependent on levels of physical activity after waking. J Hypertens. 2002;20: $865-870$.

48. Wizner B, Dechering DG, Thijs L, et al. Short-term and long-term repeatability of the morning blood pressure in older patients with isolated systolic hypertension. J Hypertens. 2008;26:1328-1335.

49. Eguchi K, Hoshide S, Hoshide Y, Ishikawa S, Shimada K, Kario K. Reproducibility of ambulatory blood pressure in treated and untreated hypertensive patients. J Hypertens. 2010;28:918-924.

50. Cheng H-MH, Wu C-LC, Sung S-HS, et al. Prognostic utility of morning blood pressure surge for 20-year all-cause and cardiovascular mortalities: results of a community-based study. J Am Heart Assoc. 2017;6:e007667.

51. Verdecchia P, Angeli F, Mazzotta G, et al. Day-night dip and earlymorning surge in blood pressure in hypertension: prognostic implications. Hypertension. 2012;60:34-42.

52. Fujiwara T, Tomitani N, Sato K, Okura A, Suzuki N, Kario K. The relationship between a blunted morning surge and a reversed nocturnal blood pressure dipping or "riser" pattern. J Clin Hypertens. 2017;19:1108-1114

53. Bombelli M, Fodri D, Toso E, et al. Relationship among morning blood pressure surge, 24-hour blood pressure variability, and cardiovascular outcomes in a white population. Hypertension. 2014;64: 943-950.

54. Marfella R, Siniscalchi M, Portoghese M, et al. Morning blood pressure surge as a destabilizing factor of atherosclerotic plaque: role of ubiquitin-proteasome activity. Hypertension. 2007;49:784-791.

55. Maeda K, Yasunari K, Watanabe T, Nakamura M. Oxidative stress by peripheral blood mononuclear cells is increased in hypertensives with an extreme-dipper pattern and/or morning surge in blood pressure. Hypertens Res. 2005;28:755-761.

56. McMullan CJ, Yano Y, Bakris GL, Kario K, Phillips RA, Forman JP. Racial impact of diurnal variations in blood pressure on cardiovascular events in chronic kidney disease. J Am Soc Hypertens. 2015;9: 299-306.

57. Pierdomenico SD, Pierdomenico AM, Cuccurullo F. Morning blood pressure surge, dipping, and risk of ischemic stroke in elderly patients treated for hypertension. Am J Hypertens. 2014;27:564-570.

58. Pierdomenico SD, Pierdomenico AM, Di Tommaso R, et al. Morning blood pressure surge, dipping, and risk of coronary events in elderly treated hypertensive patients. Am J Hypertens. 2016;29: 39-45.

59. Pierdomenico SD, Pierdomenico AM, Coccina F, Lapenna D, Porreca E. Prognostic value of nondipping and morning surge in elderly treated hypertensive patients with controlled ambulatory blood pressure. $\mathrm{Am} \mathrm{J}$ Hypertens. 2017;30:159-165.

60. Li Y, Thijs L, Hansen TW, et al. Prognostic value of the morning blood pressure surge in 5645 subjects from 8 populations. Hypertension. 2010;55:1040-1048.

61. Sheppard JP, Hodgkinson J, Riley R, Martin U, Bayliss S, McManus RJ. Prognostic significance of the morning blood pressure surge in clinical practice: a systematic review. Am J Hypertens. 2015;28:30-41.

62. Xie J-C, Yan H, Zhao Y-X, Liu X-Y. Prognostic value of morning blood pressure surge in clinical events: a meta-analysis of longitudinal Studies. J Stroke Cerebrovasc Dis. 2015;24:362-369. 
63. Parati G, Bilo G, Redon J. Morning and smooth 24-h ambulatory blood pressure control is not achieved in general practice. J Hypertens. 2013;31:616-623.

64. Parati G, Stergiou G, O’Brien E, et al. European Society of Hypertension practice guidelines for ambulatory blood pressure monitoring. J Hypertens. 2014;32:1359-1366.

65. Bilo G, Koch W, Hoshide S, Parati G. Efficacy of olmesartan/amlodipine combination therapy in reducing ambulatory blood pressure in moderate-to-severe hypertensive patients not controlled by amlodipine alone. Hypertens Res. 2014;37:836-844.

66. Eguchi K, Kario K, Hoshide Y, et al. Comparison of valsartan and amlodipine on ambulatory and morning blood pressure in hypertensive patients. Am J Hypertens. 2004;17:112-117.

67. Parati G, Bilo G, Redon J, SURGE Steering Committee. The effects of telmisartan alone or with hydrochlorothiazide on morning and 24-h ambulatory BP control: results from a practice-based study (SURGE 2). Hypertens Res. 2013;36:322-327.

68. Zhao $\mathrm{P}, \mathrm{Xu} \mathrm{P}$, Wan $\mathrm{C}$, Wang Z. Evening versus morning dosing regimen drug therapy for hypertension. In Zhao P, editor. Cochrane Database of Systematic Reviews. Chichester: John Wiley \& Sons, Ltd; 2011:CD004184.

69. Kario K, Hoshide S, Shimizu M, et al. Effect of dosing time of angiotensin II receptor blockade titrated by self-measured blood pressure recordings on cardiorenal protection in hypertensives: the Japan Morning Surge-Target Organ Protection (J-TOP) study. J Hypertens. 2010;28:1574-1583.

70. Acelajado MC, Pisoni R, Dudenbostel T, Oparil S, Calhoun DA, Glasser SP. Both morning and evening dosing of nebivolol reduces trough mean blood pressure surge in hypertensive patients. J Am Soc Hypertens. 2012;6:66-72.
71. Denardo SJ, Gong Y, Cooper-DeHoff RM, et al. Effects of verapamil sr and atenolol on 24-hour blood pressure and heart rate in hypertension patients with coronary artery disease: an international verapamil sr-trandolapril ambulatory monitoring substudy. PLoS One. 2015;10:e0122726.

72. Eguchi K, Kario K, Shimada K. Comparison of candesartan with lisinopril on ambulatory blood pressure and morning surge in patients with systemic hypertension. Am J Cardiol. 2003;92:621-624.

73. Kwon H-MM, Shin J-WW, Lim J-SS, Hong Y-HH, Lee Y-SS, Nam H. Comparison of the effects of amlodipine and losartan on blood pressure and diurnal variation in hypertensive stroke patients: a prospective, randomized, double-blind, comparative parallel study. Clin Ther. 2013;35:1975-1982.

74. Mizuno H, Hoshide S, Fukutomi M, Kario K. Differing effects of aliskiren/amlodipine combination and high-dose amlodipine monotherapy on ambulatory blood pressure and target organ protection. J Clin Hypertens. 2016;18:70-78.

75. Hermida RC, Ayala DE, Mojón A, Fernández JR. Decreasing sleeptime blood pressure determined by ambulatory monitoring reduces cardiovascular risk. J Am Coll Cardiol. 2011;58:1165-1173.

76. Kasiakogias A, Tsioufis C, Thomopoulos C, et al. Evening versus morning dosing of antihypertensive drugs in hypertensive patients with sleep apnoea: a cross-over study. J Hypertens. 2015;33:393-400.

77. Zappe DH, Crikelair N, Kandra A, Palatini P. Time of administration important? Morning versus evening dosing of valsartan. J Hypertens. 2015;33:385-392.

78. Rakugi H, Kario K, Enya K, Sugiura K, Ikeda Y. Effect of azilsartan versus candesartan on morning blood pressure surges in Japanese patients with essential hypertension. Blood Press Monit. 2014;19:164-169.
Integrated Blood Pressure Control

\section{Publish your work in this journal}

Integrated Blood Pressure Control is an international, peer-reviewed open-access journal focusing on the integrated approach to managing hypertension and risk reduction. Treating the patient and comorbidities together with diet and lifestyle modification and optimizing healthcare resources through a multidisciplinary team approach constitute key

\section{Dovepress}

features of the journal. This journal is indexed on American Chemical Society's Chemical Abstracts Service (CAS). The manuscript management system is completely online and includes a very quick and fair peerreview system, which is all easy to use. Visit http://www.dovepress.com/ testimonials.php to read real quotes from published authors.

Submit your manuscript here: https://www.dovepress.com/integrated-blood-pressure-control-journal 